\title{
Avaliação do impacto causado pela disponibilidade de 17ß-estradiol livre ou complexado à $\beta$-ciclodextrina no ambiente aquático sobre Oreochromis niloticus (tilápia)
}

\author{
[Impact evaluation caused by disponibility of free and complexed $17 \beta$-estradiol into cyclodextrin in the \\ aquatic environment in tilapia (Oreochromis niloticus)] \\ M.C.G. Silva, S.C.B.L. Silva, T.P. Santos, P.R.L. Soares, A.L.C. Andrade, \\ M.R.S. Cadena, P.G. Cadena* \\ Universidade Federal Rural de Pernambuco - Recife, PE
}

\begin{abstract}
RESUMO
Foram avaliados os efeitos tóxicos do hormônio $17 \beta$-estradiol $\left(\mathrm{E}_{2}\right)$ livre e complexado à $\beta$-ciclodextrina $(\mathrm{CD})$ sobre o comportamento e a fisiologia de tilápia (Oreochromis niloticus). Os peixes foram observados por 30 dias, em dois estágios do desenvolvimento (alevino e juvenil), pelo método ad libitum, para a confecção de um etograma. Posteriormente, juvenis foram divididos em três grupos: controle e expostos ao $\mathrm{E}_{2}(10 \mathrm{ng} / \mathrm{L})$ livre e complexado à $\beta$-ciclodextrina $\left(\beta-\mathrm{CD}: \mathrm{E}_{2}\right)$ por 90 dias. Foram avaliados o comportamento pelo método de varredura instantânea, o consumo de ração, o ganho de peso e a mortalidade em diferentes intervalos. Os alevinos e os juvenis apresentaram frequências de exibição comportamentais diferentes $(\mathrm{P}<0,05)$ nos eventos: Afastar $(4,7 \pm 1,3$ e 3,6 $\pm 0,6 \%)$ e Ondulação de repulsão $(2,3 \pm 0,9$ e 1,3 $\pm 1,0 \%)$. Os juvenis expostos ao complexo $\beta-\mathrm{CD}: \mathrm{E}_{2}$ apresentaram aumento $(\mathrm{P}<0,05)$ na exibição dos comportamentos agressivos, como Afastar, Ataque caudal, Confronto prolongado, Perseguição, Fuga, e menor mortalidade, quando comparados ao grupo exposto ao $E_{2}$ livre e controle. Pode-se concluir que a complexação do $E_{2}$ com a $\beta$-CD alterou a toxicidade do $\mathrm{E}_{2}$, pois promoveu um aumento na frequência de exibição dos comportamentos agressivos e interferiu na mortalidade dos animais.
\end{abstract}

Palavras-chave: comportamento animal, etograma, complexo de inclusão, sobrevivência

\begin{abstract}
Toxic effects of free and complexed 17 $\beta$-estradiol (E2) hormone into $\beta$-cyclodextrin (CD) on the behavior and physiology of tilapia (Oreochromis niloticus) were evaluated. The fish were observed for 30 days in two stages of development (fingerling and juvenile) by the ad libitum method to make an ethogram. After this, juveniles were divided into three groups: control and exposed to free $E_{2}(10 \mathrm{ng} / \mathrm{L})$ and complexed into $\beta$-cyclodextrin $(\beta$ $\left.C D: E_{2}\right)$ for 90 days. The behavior was evaluated through scan sampling method, feed intake, body mass and mortality at different intervals. The fingerlings and juveniles showed behavioral patterns with different display frequencies $(P<0.05)$ for events: Move Away $(4.7 \pm 1.3$ and $3.6 \pm 0.6 \%)$ and Waving Repulsion $(2.3 \pm 0.9$ and $1.3 \pm 1.0 \%)$. The juveniles exposed to $\beta-C D: E_{2}$ complex showed a significant increase $(P<0.05)$ in the frequency of display of aggressive behaviors as Move Away, Caudal Attack, Clash Extended, Chase, Escape and decrease of mortality when compared to group exposed to free $E_{2}$ and control. In conclusion, complexation of $E_{2}$ into $\beta$ $C D$ modified $E_{2}$ toxicity, because it promoted an increase in the frequency of display of aggressive behaviors and it affected the mortality of animals.
\end{abstract}

Keywords: animal behavior, ethogram, inclusion complex, mortality

\section{INTRODUÇÃO}

Devido às características químicas dos hormônios esteroides como a hidrofobicidade, a indústria farmacêutica vem utilizando

Recebido em 22 de agosto de 2016

Aceito em 22 de fevereiro de 2017

*Autor para correspondência (corresponding author)

E-mail: pabyton.cadena@ufrpe.br substâncias químicas para superar essa limitação, sendo possível complexar esses hormônios a ciclodextrinas (CDs) (Oishi et al., 2008). CDs são oligossacarídeos cíclicos, constituídos por um número variável de unidades de glicose, unidas por ligações glicosídicas $\alpha-(1,4)$. São 
estruturas troncocônica e se caracterizam por possuírem cavidade de natureza apolar, que contrasta com o exterior hidrofílico (Del Valle, 2004; Brewster e Loftsson, 2007). Devido a isso, podem formar complexos de inclusão hospedando moléculas hidrofóbicas em seu interior. São utilizadas com o objetivo de melhorar as características farmacocinéticas de substâncias, aumentando a biodisponibilidade, a estabilidade e diminuindo a volatilidade (Brewster e Loftsson, 2007). Seus efeitos tóxicos são conhecidos em humanos devido a sua ampla utilização na farmacêutica, na indústria alimentícia e de cosméticos, onde apresenta nefrotoxicidade por via parenteral, mas não foram relatados efeitos tóxicos por via oral (Del Valle, 2004; Brewster e Loftsson, 2007). Porém, os efeitos desses complexos de inclusão como poluente ambiental e seus efeitos em animais são pouco descritos na literatura. Adicionalmente, hormônios esteroides, quando complexados a CDs, podem se apresentar como disruptores endócrinos (DEs). Os DEs são persistentes e passíveis de sofrerem biomagnificação, principalmente em animais dulciaquícolas (Wu et al., 2016), que são sensíveis a baixas concentrações em níveis subletais. Nesses casos, foram observadas principalmente alterações comportamentais e reprodutivas, que podem levar a desequilíbrios populacionais em ambientes aquáticos (Santos et al., 2016; Denslow e Sepúlveda, 2008). Isso destaca a necessidade de maiores estudos dos efeitos de toxicidade decorrente da disposição desses complexos de inclusão entre CDs e fármacos na biota aquática.

Contaminantes naturais e sintéticos presentes em concentrações na faixa de $\mathrm{pg} / \mathrm{L}$ a $\mathrm{ng} / \mathrm{L}$ apresentam um risco à saúde dos ecossistemas, pois existe uma carência na avaliação dos seus efeitos fisiológicos, no conhecimento de suas fontes, no comportamento no ambiente e nos níveis tóxicos de concentração (Fernandes et al., 2011). Entre esses contaminantes, os DEs têm despertado atenção científica por causa de sua distribuição generalizada e de seus efeitos potencialmente nocivos para a saúde (Wu et al., 2016). Estes são capazes de mimetizar os efeitos moleculares de hormônios endógenos ou de causar desequilíbrio hormonal, por interferirem na sua síntese, na secreção, no transporte, no metabolismo e na excreção, consequentemente, na reprodução, no desenvolvimento e no comportamento de espécies animais (Denslow e Sepúlveda, 2008). Estrógenos como o 17ßestradiol $\left(E_{2}\right)$ regulam e sustentam o desenvolvimento sexual feminino e suas funções reprodutivas, pois deles depende a formação dos ovários nas fêmeas (Guiguen et al., 2010). Esse hormônio possui conformações reconhecidas pelos receptores e, portanto, é considerado responsável pela maioria dos efeitos disruptores, quando dispostos nos efluentes, como redução do tamanho das gônadas, feminização de peixes machos e problemas no desenvolvimento do sistema imune (Tilton et al., 2005; McLachlan et al., 2006; Seemann et al., 2015).

Anteriormente, Santos et al. (2016) haviam estudado os efeitos de DEs presentes em contraceptivos orais combinados sobre $\mathrm{o}$ comportamento agressivo de Betta splendes. Hoje, o objetivo deste trabalho foi avaliar os efeitos tóxicos do 17ß-estradiol livre e complexado à ciclodextrina sobre o comportamento e a fisiologia de tilápia-do-nilo (Oreochromis niloticus). Trata-se de um peixe de ampla aceitação pelo mercado consumidor, com produção mundial em 2014 de quase quatro milhões de toneladas (Species..., 2016), o qual apresenta agressividade natural, sendo assim um bom modelo animal para avaliar alterações comportamentais provenientes da exposição ao $17 \beta$-estradiol livre e complexado à CDs.

\section{MATERIAL E MÉTODOS}

O estudo foi realizado no Laboratório de Ecofisiologia e Comportamento Animal - Leca, da Universidade Federal Rural de Pernambuco UFRPE. O 17 $\beta$-estradiol e a $\beta$-ciclodextrina (SIGMA, EUA) e os outros reagentes utilizados foram de grau analítico. As tilápias (Oreochromis niloticus) foram obtidas da Estação de Aquicultura Continental Professor Johei Koike - Departamento de Pesca da UFRPE. Os protocolos realizados neste trabalho foram aprovados pelo Comitê de Ética no Uso de Animais - Ceua/UFRPE, licença 001/2014. No laboratório, os animais foram aclimatados por 15 dias, sendo acondicionados em aquários aerados, em temperatura ambiente $\left(27^{\circ} \mathrm{C}\right)$, sempre com densidade de um animal para aproximadamente 12 litros de água e ciclo de luz natural. $O$. niloticus foram alimentados ad libitum, com ração comercial extrusada $(45 \%$ de proteína bruta) uma vez ao dia, com quantidade crescente 
de acordo com o crescimento do animal. Em relação aos parâmetros abióticos, o $\mathrm{pH}$ e o oxigênio dissolvido (OD) foram aferidos semanalmente com potenciômetro e medidor de OD portátil, durante todos os experimentos. Foram utilizados kits comerciais da marca Alcon Labtest, para nitrito, amônia tóxica e amônia total.

Para a construção do etograma e análise comportamental comparativa, 14 animais, sendo sete alevinos $(9,7 \pm 1,1 \mathrm{~g}, 3,8 \pm 0,2 \mathrm{~cm})$ e sete juvenis $(38,0 \pm 1,3 \mathrm{~g}, \quad 14,3 \pm 0,6 \mathrm{~cm})$ foram separados e alocados em aquários de $85 \mathrm{~L}$ cada. O etograma foi construído pelo método ad libitum (Altmann, 1974) por 30 dias, a uma distância preestabelecida $(2,5 \mathrm{~m})$, de modo a não influenciar na exibição comportamental. Foram utilizados indivíduos em estágios de desenvolvimentos distintos (alevinos e juvenis) para que houvesse a possibilidade de comparação do padrão comportamental em diferentes estágios de desenvolvimento. A coleta dos dados comportamentais com o etograma foi realizada pelo método de scan sampling (Altmann, 1974), por 30 minutos, com um minuto de observação e um minuto de intervalo.

Para os testes de exposição química, os animais foram submetidos a concentrações dos hormônios em teste de toxicidade crônica, em sistema semiestático por 90 dias, e divididos em três grupos de peixes juvenis alocados em aquários de $85 \mathrm{~L}$, com sete animais cada, sendo quatro machos e três fêmeas por grupo, identificados mediante sexagem manual, por meio do exame da papila urogenital (Ávila e Romagosa, 2005). Os grupos foram: controle (grupo C) sem adição do hormônio, 17 $\beta$-estradiol (grupo E) na concentração de $10 \mathrm{ng} / \mathrm{L}$, com adição de solução aquosa do complexo de inclusão $\beta$-ciclodextrina - 17 $\beta$-estradiol $(\beta$ CD:E2), na proporção de $1: 1 \mathrm{em} \mathrm{mol} / \mathrm{L}$, mantendo-se a concentração de E2 em 10ng/L (grupo BE). A técnica de coprecipitação (Del

Valle, 2004; Oishi et al., 2008) foi utilizada para formação dos complexos de inclusão entre a $\beta$ $\mathrm{CD}$ e o hormônio $\mathrm{E}_{2}$ em meio aquoso. $\mathrm{A} \beta-\mathrm{CD}$ foi primeiramente solubilizada em água, e o $E_{2}$ dissolvido em etanol (10\%), com posterior evaporação do álcool. A solução de $\mathrm{E}_{2}$ foi adicionada sob agitação à solução de CD para permitir a complexação deles. Os complexos de inclusão formados foram armazenados sob refrigeração $\left(4^{\circ} \mathrm{C}\right)$ para posterior uso. Houve renovação completa da água dos aquários semanalmente e reposição das concentrações hormonais utilizadas.

Durante os testes de exposição química com os animais, foi mensurado o comportamento animal conforme descrito anteriormente. Os dados comportamentais foram agrupados a cada 30 dias, somando três períodos de exposições distintos (zero a 30, 30 a 60 e 60 a 90 dias). O consumo médio de ração foi avaliado diariamente conforme descrito por Zuanon et al. (2009). Após a determinação do tempo para a saciedade, os restos de ração foram removidos dos aquários para evitar contaminação. Para a avaliação do ganho de peso, foi mesurada a diferença entre os pesos inicial e final dos animais. A mortalidade foi verificada diariamente. Os resultados foram expressos na forma de média e desvio-padrão e comparados por análise de variância simples (One Way ANOVA). Quando o resultado foi significativo, as médias foram comparadas pelo teste de Tukey, utilizando-se o software Origin Pro Academic 2015 (Origin Lab. Northampton, MA USA). As diferenças foram consideradas significativas quando $\mathrm{P}<0,05$.

\section{RESULTADOS}

Para os parâmetros físico-químicos, a temperatura da água nos aquários não apresentou variação maior que $0,5^{\circ} \mathrm{C}$ durante o experimento e o $\mathrm{pH}$ apresentou faixa entre 6,7 e 7,3. Para o $\mathrm{OD}$, os valores sempre foram superiores a $9 \mathrm{mg} / \mathrm{L}$. Para o nitrito, foram encontrados valores de $0,25 \mathrm{mg} / \mathrm{L}$, e não foram observados valores de nitrato. Para amônia tóxica, foram encontrados valores inferiores a $1 \mathrm{mg} / \mathrm{L}$.

Entre os dados obtidos a partir do método ad libitum (Altmann, 1974) para a elaboração do etograma, foram identificados 24 comportamentos, agrupados nas categorias: Alimentação, Locomoção, Social e Resposta ao estresse, que podem ser observados na Tab. 1 . 
Tabela 1. Etograma dos alevinos e juvenis de tilápia (Oreochromis niloticus)

\begin{tabular}{|c|c|c|}
\hline \multirow{2}{*}{ Evento Sigla } & \multicolumn{2}{|r|}{ Descrição } \\
\hline & & Categoria Alimentação \\
\hline Forragear & FO & $\begin{array}{l}\mathrm{O} \text { animal posiciona-se quase de maneira vertical, próximo ao substrato, } \\
\text { procurando alimento. }\end{array}$ \\
\hline Procurar & PR & $\begin{array}{l}\mathrm{O} \text { animal se mantém próximo à lâmina d'água e nada obliquamente, } \\
\text { procurando alimento }\end{array}$ \\
\hline Capturar rápido & $\mathrm{CR}$ & $\begin{array}{l}\text { O animal nada até a lâmina d'água, captura o alimento na superfície e volta } \\
\text { subitamente ao fundo do aquário, podendo repetir diversas vezes. }\end{array}$ \\
\hline Capturar prolongado & $\mathrm{CP}$ & $\begin{array}{l}\text { O peixe permanece se alimentando na superfície da água, fazendo } \\
\text { movimentos rápidos com a boca em direção ao alimento, a fim de ingeri-lo. }\end{array}$ \\
\hline Regurgitar & RG & $\begin{array}{l}\text { O animal, depois de saciado, ingere o alimento, mastiga e lança as partículas } \\
\text { novamente na água pela boca. }\end{array}$ \\
\hline \multicolumn{3}{|r|}{ Categoria Social } \\
\hline Afugentar & AG & $\begin{array}{l}\text { O animal nada em direção ao oponente rapidamente, podendo ou não haver } \\
\text { contato, provocando o afastamento do outro. }\end{array}$ \\
\hline Afugentar prolongado & AP & $\begin{array}{l}\text { O animal (1) passa a ocupar uma boa parte da área do aquário e afugenta os } \\
\text { outros animais (2), que ficam restritos a um pequeno espaço. }\end{array}$ \\
\hline Afastar & AT & $\begin{array}{l}\text { O animal (1) se afasta com um movimento curto do oponente (2) após sofrer } \\
\text { um ataque. }\end{array}$ \\
\hline Exibição lateral & EL & $\begin{array}{l}\text { O peixe se aproxima lateralmente do outro, abre a boca, mas não ocorre } \\
\text { contato físico com o oponente. Esse comportamento pode culminar em um } \\
\text { ataque. }\end{array}$ \\
\hline Ataque lateral & $\mathrm{AL}$ & $\begin{array}{l}\text { O peixe abre a boca e percorre com ela o corpo do oponente, fechando-a no } \\
\text { momento do contato. O ataque pode ocorrer nas laterais medianas do corpo, } \\
\text { no dorso, nas nadadeiras do outro animal. }\end{array}$ \\
\hline Ataque rápido & AR & $\begin{array}{l}\text { O peixe nada próximo à lâmina d'água e desce rapidamente, fazendo um } \\
\text { ataque ao oponente. }\end{array}$ \\
\hline Ataque caudal & $\mathrm{AC}$ & $\begin{array}{l}\text { O animal nada próximo ao oponente, fechando a boca quando esta entra em } \\
\text { contato com a nadadeira caudal do adversário. }\end{array}$ \\
\hline Ataque frontal & $\mathrm{AF}$ & $\begin{array}{l}\text { Dois peixes sobrepõem suas mandíbulas e um deles empurra o outro, ou } \\
\text { ambos se empurram mutuamente em movimentos rápidos. }\end{array}$ \\
\hline Confronto prolongado & $\mathrm{CG}$ & Dois animais realizam ataques mútuos com movimento rotatório. \\
\hline Ondulação & $\mathrm{ON}$ & $\begin{array}{l}\text { O peixe ondula o corpo no sentido anteroposterior quando está próximo ao } \\
\text { oponente. }\end{array}$ \\
\hline $\begin{array}{l}\text { Ondulação de } \\
\text { repulsão }\end{array}$ & OR & $\begin{array}{l}\text { O peixe agredido exibe ondulações rápidas e intensas com o corpo que } \\
\text { levam à repulsão do peixe agressor. }\end{array}$ \\
\hline Perseguição & PE & $\begin{array}{l}\text { O peixe segue o oponente enquanto este foge, podendo culminar em ataque } \\
\text { do peixe agressor. }\end{array}$ \\
\hline Fuga & FU & O peixe se afasta do oponente que o persegue ou ataca. \\
\hline Fuga prolongada & FR & $\begin{array}{l}\text { O animal se afasta do oponente, ficando restrito a um pequeno espaço por } \\
\text { longo período de tempo. }\end{array}$ \\
\hline \multicolumn{3}{|r|}{ Categoria Locomoção } \\
\hline Nadar lento & NL & $\begin{array}{l}\text { O animal movimenta a nadadeira caudal lentamente, a fim de que seu } \\
\text { deslocamento ocorra também de modo lento. }\end{array}$ \\
\hline Nadar rápido & NR & $\begin{array}{l}\text { O animal movimenta sua nadadeira caudal rapidamente, fazendo com que } \\
\text { ele se desloque mais rápido no corpo d'água. Esse comportamento pode ser } \\
\text { seguido por qualquer ataque. }\end{array}$ \\
\hline Nadar junto & NJ & $\begin{array}{l}\text { Três ou mais peixes nadam na mesma direção, apresentando comportamento } \\
\text { de cardume, podendo permanecer assim por vários minutos. }\end{array}$ \\
\hline Ficar parado & FP & O peixe permanece parado. \\
\hline \multicolumn{3}{|r|}{ Categoria Resposta ao estresse } \\
\hline Respiração aérea & RA & eixe utiliza a respiração aérea mesmo sob condições de aeração. \\
\hline
\end{tabular}


$\mathrm{Na}$ Fig. 1, é possível observar os eventos comportamentais dos alevinos (A) e juvenis $(\mathrm{J})$ de $O$. niloticus, em que, na categoria Social, os mesmos comportamentos foram apresentados pelos dois grupos, mas com frequências de exibição diferentes $(\mathrm{P}<0,05)$ para os eventos: Afastar (A 4,7 71,3 e J 3,6 $\pm 0,6 \%$ ) e Ondulação de

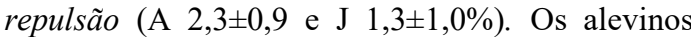
apresentaram maior frequência de exibição nesses comportamentos. Na categoria Locomoção, o comportamento Nadar rápido (A 9,2 $\pm 2,8$ e J $3,0 \pm 1,6 \%$ ) foi mais observado nos alevinos em comparação aos juvenis. Em relação às outras categorias de Alimentação e Resposta ao estresse, não houve diferença significativa $(P>0,05)$ entre os grupos estudados.

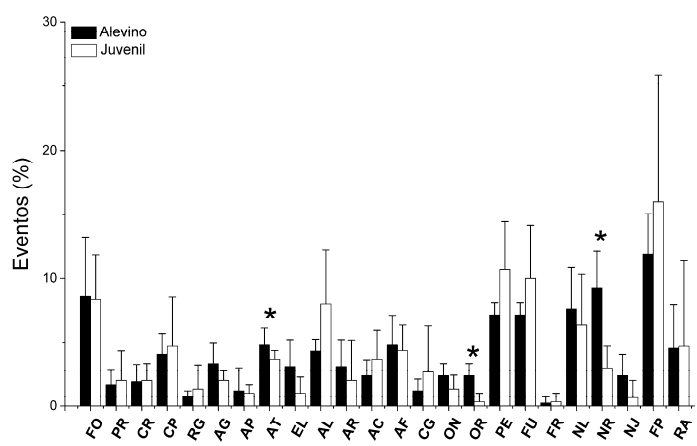

Figura 1. Eventos comportamentais exibidos por $O$. niloticus alevinos e juvenis. Siglas: FO - Forragear; PR - Procurar; CR - Capturar rápido; CP - Capturar prolongado; RG - Regurgitar; AG - Afugentar; AP Afugentar prolongado; AT - Afastar; EL - Exibição lateral; $\mathrm{AL}$ - Ataque lateral; $\mathrm{AR}$ - Ataque rápido; $\mathrm{AC}$ - Ataque caudal; AF - Ataque frontal; CG - Confronto prolongado; ON - Ondulação; OR - Ondulação de repulsão; PE - Perseguição; FU - Fuga; FR - Fuga prolongada; NL - Nadar lento; NR - Nadar rápido; NJ - Nadar junto; FP - Ficar parado; RA - Respiração aérea. *Médias com diferença significativa $(\mathrm{P}<0,05)$ pelo teste de Tukey.

$\mathrm{Na}$ Tab. 2a, são observados os resultados das frequências de exibição dos comportamentos de juvenis de $O$. niloticus sob exposição ao $\mathrm{E}_{2}$ e à $\beta$ $\mathrm{CD}: \mathrm{E}_{2}$ e ao grupo controle, no intervalo de zero a 30 dias. Na categoria Alimentação, foi observado um aumento (Tab. 2a) significativo $(\mathrm{P}<0,05)$ na frequência de exibição do comportamento Procurar no grupo BE em relação ao grupo E. $\mathrm{Na}$ categoria Social, houve um aumento significativo $(\mathrm{P}<0,05)$ na exibição do comportamento Afugentar prolongado no grupo $\mathrm{E}$ em relação aos grupos $\mathrm{BE}$ e C. Ainda, houve um aumento (Tab. $2 a)$ significativo $(\mathrm{P}<0,05)$ na frequência de exibição dos comportamentos agressivos Afastar, Ondulação, Perseguição, Fuga, Fuga prolongada no grupo BE; esse último comportamento, apenas observado neste grupo. Para a categoria Locomoção, também foi observado um aumento (Tab. 2a) significativo $(\mathrm{P}>0,05)$ da exibição do comportamento Nadar Rápido no grupo BE. Na Tab. 2b, é possível observar os comportamentos exibidos pelos peixes sob exposição ao $E_{2}$, à $\beta-C D: E_{2}$ e ao grupo controle, no intervalo de 30 a 60 dias. $\mathrm{Na}$ categoria Alimentação, foi observado um aumento (Tab. 2b) significativo $(\mathrm{P}<0,05)$ na frequência de exibição do comportamento Procurar no grupo $\mathrm{BE}$ em relação aos grupos E e C. Para a categoria Social, verificou-se um aumento significativo $(\mathrm{P}<0,05)$ para o grupo $\mathrm{BE}$ nos comportamentos: Afastar, Exibição lateral, Ataque caudal, Ataque frontal, Confronto prolongado, Ondulação, Ondulação de repulsão, Perseguição, Fuga e Fuga prolongada. Na categoria Locomoção, o grupo $\mathrm{BE}$ apresentou um aumento $(\mathrm{P}<0,05)$ na exibição dos comportamentos Nadar lento e Nadar rápido (Tab. 2b).

$\mathrm{Na}$ Tab. 2c, são observados os resultados da exibição comportamental dos juvenis no intervalo de 60 a 90 dias de experimento. Não houve eventos significativos $(\mathrm{P}>0,05)$ nas categorias Alimentação e Resposta ao estresse. Foram observadas diferenças significativas $(\mathrm{P}<0,05)$ em alguns comportamentos das categorias Social e Locomoção, com aumento de frequência (Tab. 2c) para o grupo BE em Ataque frontal, Nadar lento e Nadar rápido. Ainda, os animais do grupo $\mathrm{BE}$ não apresentaram a exibição do comportamento Afastar $(\mathrm{P}<0,05)$. Adicionalmente, foi observado o escurecimento corporal dos animais submissos durante os experimentos. O consumo médio de ração entre os grupos não apresentou diferença significativa $(\mathrm{P}>0,05)$, pois todo o alimento foi consumido. Para avaliação do ganho de peso, também não foram observadas diferenças significativas $(\mathrm{P}>0,05)$ entre a comparação do grupo $\mathrm{C} \quad(28,9 \pm 9,3 \mathrm{~g}$ e $42,5 \pm 10,5 \mathrm{~g})$, grupo $\mathrm{E}(25,0 \pm 6,4 \mathrm{~g}$ e $34,6 \pm 12,0 \mathrm{~g}) \mathrm{e}$ grupo $\mathrm{BE}(31,0 \pm 9,0 \mathrm{~g}$ e $39,4 \pm 8,2 \mathrm{~g})$ no intervalo de zero e 90 dias. Essa diferença não significativa $(\mathrm{P}>0,05)$ entre a morfometria nos grupos $\mathrm{C}(12,1 \pm 1,8$ e $13,4 \pm 1,4), \mathrm{E}(10,9 \pm 0,8 \mathrm{e}$ $12,2 \pm 1,7)$ e $\operatorname{BE}(12,5 \pm 1,7$ e $13,1 \pm 1,8)$ também foi observada no mesmo intervalo de tempo. Em relação à taxa de mortalidade, foram encontrados os valores de C 42,9\%, E 28,6\% e BE 14,3\%, respectivamente, após 90 dias. 
Tabela 2. Eventos comportamentais de O. niloticus expostos a hormônios livres e complexados à ciclodextrina em 30 (A), 60 (B) e 90 (C) dias de exposição. Para os eventos exibidos: FO - Forragear; PR - Procurar; CR - Capturar rápido; CP - Capturar prolongado; RG - Regurgitar; AG - Afugentar; AP Afugentar prolongado; AT - Afastar; EL - Exibição lateral; AL - Ataque lateral; AR - Ataque rápido; AC - Ataque caudal; AF - Ataque frontal; CG - Confronto prolongado; ON - Ondulação; OR - Ondulação de repulsão; PE - Perseguição; FU - Fuga; FR - Fuga prolongada; NL - Nadar lento; NR - Nadar rápido; NJ Nadar junto; FP - Ficar parado; RA - Respiração aérea

\begin{tabular}{|c|c|c|c|c|c|c|c|c|c|}
\hline \multirow{2}{*}{ Eventos } & \multicolumn{3}{|c|}{ A: $0-30$ dias } & \multicolumn{3}{|c|}{ B: $30-60$ dias } & \multicolumn{3}{|c|}{ C: $60-90$ dias } \\
\hline & $\mathrm{C}$ & $\mathrm{E}$ & $\mathrm{BE}$ & $\mathrm{C}$ & $\mathrm{E}$ & $\mathrm{BE}$ & $\mathrm{C}$ & $\mathrm{E}$ & $\mathrm{BE}$ \\
\hline FO & $6,3 \pm 3,5$ & $5,7 \pm 3,9$ & $6,6 \pm 3,3$ & $1,6 \pm 1,4^{\mathrm{a}}$ & $0,7 \pm 0,9^{\mathrm{a}}$ & $4,8 \pm 1,9^{b}$ & $1,6 \pm 0,5$ & $2,2 \pm 1,9$ & $2,4 \pm 0,5$ \\
\hline PR & $0,3 \pm 0,4^{\mathrm{a}}$ & $0,1 \pm 0,3^{\mathrm{ab}}$ & $1,0 \pm 0,9^{\mathrm{ac}}$ & $0,1 \pm 0,3^{\mathrm{a}}$ & $0,1 \pm 0,3^{\mathrm{a}}$ & $1,6 \pm 1,3^{b}$ & $0,0 \pm 0,0$ & $0,2 \pm 0,4$ & $0,0 \pm 0,0$ \\
\hline $\mathrm{CP}$ & $0,0 \pm 0,0$ & $0,0 \pm 0,0$ & $0,0 \pm 0,0$ & $0,0 \pm 0,0$ & $0,0 \pm 0,0$ & $0,1 \pm 0,3$ & $0,0 \pm 0,0$ & $0,0 \pm 0,0$ & $0,0 \pm 0,0$ \\
\hline RG & $0,1 \pm 0,3$ & $0,2 \pm 0,4$ & $0,3 \pm 0,4$ & $0,0 \pm 0,0$ & $0,0 \pm 0,0$ & $0,2 \pm 0,4$ & $0,0 \pm 0,0$ & $0,0 \pm 0,0$ & $0,0 \pm 0,0$ \\
\hline $\mathrm{AG}$ & $2,9 \pm 3,2$ & $2,8 \pm 3,1$ & $2,4 \pm 2,6$ & $1,0 \pm 1,7$ & $0,7 \pm 1,2$ & $1,1 \pm 1,1$ & $0,2 \pm 0,4$ & $2,6 \pm 3,1$ & $0,0 \pm 0,0$ \\
\hline EL & $0,8 \pm 0,9$ & $0,6 \pm 0,6$ & $1,2 \pm 1,2$ & $0,7 \pm 1,0^{\mathrm{a}}$ & $0,4 \pm 0,7^{\mathrm{a}}$ & $3,0 \pm 1,9^{b}$ & $0,8 \pm 0,4$ & $0,2 \pm 0,4$ & $1,4 \pm 1,5$ \\
\hline $\mathrm{AL}$ & $5,8 \pm 2,9$ & $8,2 \pm 3,8$ & $5,3 \pm 2,5$ & $5,8 \pm 2,5$ & $6,0 \pm 1,8$ & $6,0 \pm 1,5$ & $6,2 \pm 1,6$ & $6,6 \pm 2,7$ & $5,4 \pm 1,3$ \\
\hline $\mathrm{AR}$ & $8,3 \pm 3,7$ & $5,2 \pm 1,6$ & $7,0 \pm 2,7$ & $5,6 \pm 2,5$ & $5,7 \pm 2,3$ & $6,5 \pm 1,2$ & $5,6 \pm 1,1$ & $5,4 \pm 2,3$ & $6,6 \pm 1,5$ \\
\hline $\mathrm{AC}$ & $1,7 \pm 1,9^{\mathrm{a}}$ & $3,3 \pm 2,5^{\mathrm{ab}}$ & $5,0 \pm 2,3^{\mathrm{b}}$ & $1,4 \pm 2,0^{\mathrm{a}}$ & $0,6 \pm 1,0^{\mathrm{a}}$ & $4,0 \pm 1,8^{\mathrm{b}}$ & $0,2 \pm 0,4$ & $2,6 \pm 2,8$ & $2,6 \pm 1,1$ \\
\hline $\mathrm{AF}$ & $3,4 \pm 4,7^{\mathrm{a}}$ & $2,2 \pm 1,6^{\mathrm{ab}}$ & $7,9 \pm 3,2^{b}$ & $2,2 \pm 2,2^{\mathrm{a}}$ & $0,1 \pm 0,3^{\mathrm{ab}}$ & $7,3 \pm 2,9^{b}$ & $1,2 \pm 0,8^{\mathrm{a}}$ & $1,4 \pm 1,5^{\mathrm{a}}$ & $5,0 \pm 1,0^{b}$ \\
\hline FU & $8,9 \pm 2,1^{\mathrm{a}}$ & $7,3 \pm 2,9^{\mathrm{ab}}$ & $11,6 \pm 3,5^{\mathrm{ac}}$ & $5,7 \pm 2,4^{\mathrm{a}}$ & $5,3 \pm 2,2^{\mathrm{a}}$ & $9,5 \pm 3,8^{b}$ & $4,6 \pm 1,1$ & $6,8 \pm 2,1$ & $5,2 \pm 1,6$ \\
\hline FR & $0,0 \pm 0,0^{\mathrm{a}}$ & $0,0 \pm 0,0^{\mathrm{a}}$ & $0,6 \pm 0,8^{\mathrm{b}}$ & $0,2 \pm 0,4^{\mathrm{a}}$ & $0,0 \pm 0,0^{\mathrm{a}}$ & $1,0 \pm 1,0^{\mathrm{b}}$ & $0,0 \pm 0,0$ & $0,0 \pm 0,0$ & $1,0 \pm 2,2$ \\
\hline NL & $6,0 \pm 3,4$ & $6,9 \pm 4,5$ & $8,0 \pm 2,9$ & $2,6 \pm 2,1^{\mathrm{a}}$ & $3,6 \pm 1,5^{\mathrm{a}}$ & $8,0 \pm 1,8^{b}$ & $3,2 \pm 0,8^{\mathrm{a}}$ & $3,0 \pm 1,5^{\mathrm{a}}$ & $7,0 \pm 3,0^{\mathrm{b}}$ \\
\hline NR & $2,2 \pm 1,1^{\mathrm{a}}$ & $0,7 \pm 1,0^{\mathrm{a}}$ & $5,3 \pm 3,0^{b}$ & $1,1 \pm 1,1^{\mathrm{a}}$ & $0,3 \pm 0,5^{\mathrm{a}}$ & $3,5 \pm 2,5^{b}$ & $1,6 \pm 0,5^{\mathrm{a}}$ & $0,4 \pm 0,5^{\mathrm{ab}}$ & $3,6 \pm 2,8^{\mathrm{ac}}$ \\
\hline NJ & $0,0 \pm 0,0$ & $0,0 \pm 0,0$ & $0,0 \pm 0,0$ & $0,0 \pm 0,0^{\mathrm{a}}$ & $0,0 \pm 0,0^{\mathrm{b}}$ & $0,0 \pm 0,0^{\mathrm{c}}$ & $0,2 \pm 0,4$ & $0,0 \pm 0,0$ & $0,0 \pm 0,0$ \\
\hline FP & $8,1 \pm 4,4^{\mathrm{a}}$ & $23,6 \pm 7,4^{b}$ & $12,3 \pm 7,3^{\mathrm{a}}$ & $15,3 \pm 8,1$ & $25,4 \pm 14,1$ & $17,3 \pm 7,4$ & $20,6 \pm 1,1^{\mathrm{a}}$ & $8,4 \pm 3,0^{\mathrm{b}}$ & $25,4 \pm 2,8^{c}$ \\
\hline RA & $7,2 \pm 2,7$ & $7,2 \pm 3,7$ & $4,6 \pm 3,4$ & $3,5 \pm 1,7$ & $4,5 \pm 4,6$ & $6,5 \pm 2,5$ & $2,6 \pm 1,3$ & $5,4 \pm 3,2$ & $7,4 \pm 5,3$ \\
\hline
\end{tabular}

Os dados foram comparados estatisticamente dentro dos intervalos de 30 dias. Médias com diferentes letras diferem estatisticamente pelo teste de Tukey $(\mathrm{P}<0,05)$.

\section{DISCUSSÃO}

Os comportamentos observados em $O$. niloticus são característicos do gênero, devido à semelhança aos observados em outros ciclídeos, como Geophagus surinamensis (Acará-corró), Astronotus ocellatos (Apaiari) e Pterophyllum scalare (Acará-bandeira) (Carvalho, 2009). Por ser uma espécie territorialista, O. niloticus apresentou uma hierarquia de dominância e submissão estabelecida por confrontos entre os indivíduos. Tais comportamentos agonísticos foram decorrentes de agrupamentos sociais, nos quais foi observada uma hierarquia de dominância e submissão em que os animais maiores geralmente são dominantes, e os menores submissos (Merighe et al., 2004). Em relação à comparação dos comportamentos exibidos entre alevinos e juvenis, com base no etograma desenvolvido, foi observada uma maior frequência de comportamentos ativos nos alevinos, o que pode estar relacionado à maior atividade locomotora destes. Larvas e alevinos de peixes diurnos (como a tilápia) apresentam maior atividade metabólica nessas fases de desenvolvimento (Vera et al., 2009), o que poderia justificar essa diferença observada no presente estudo.

Em relação aos estudos de exposição química, os parâmetros abióticos foram mensurados para comprovar que as modificações nos parâmetros biológicos analisados foram decorrentes da exposição ao estradiol livre $\left(\mathrm{E}_{2}\right)$ e complexado à ciclodextrina $\left(\beta-C D: E_{2}\right)$ e não decorrentes de variações nos parâmetros físico-químicos da água. Esses resultados obtidos, de acordo com Kubitza (2000), estão na faixa ideal de conforto para a espécie. Diante disto, as alterações comportamentais exibidas pelos animais estão relacionadas à exposição química aos hormônios. Os resultados produzidos no presente estudo demonstraram que o $E_{2}$ livre assim como a $\beta$ $\mathrm{CD}: \mathrm{E}_{2}$ interferiram no comportamento animal, alterando a exibição dos comportamentos ao longo do tempo nos animais juvenis de $O$. 
niloticus. Em relação ao efeito do $\mathrm{E}_{2}$ complexado à $\beta-C D$, foi observado um aumento na exibição de comportamentos agressivos (Tab. 2) no grupo $\mathrm{BE}$ comparado aos grupos $\mathrm{E}$ e $\mathrm{C}$, o que pode provocar maior estresse entre os indivíduos. Essas respostas biológicas ao estresse, provocadas por poluentes, podem ser utilizadas para identificar sinais iniciais de danos aos peixes (Lupi et al., 2007). De acordo com Brewster e Loftsson (2007), as CDs apresentam baixa toxicidade, mas, devido ao fato de alterarem as propriedades físico-químicas das moléculas complexadas, podem também alterarlhes a toxicidade. Isso foi observado no presente trabalho, pois houve diferenças significativas na exibição dos comportamentos $(\mathrm{P}<0,05)$ entre os grupos $\mathrm{BE}$ e E. Em peixes, existem poucos estudos sobre os efeitos tóxicos de moléculas complexadas à CDs. Estudo utilizando a hidropropil- $\beta$-ciclodextrina não complexada foi realizado em Danio rerio. O desenvolvimento de larvas foi afetado com concentrações acima de $1 \%(\mathrm{p} / \mathrm{v})$, e testes de toxicidade com peixes adultos não foram relatados (Maes et al., 2012). É possível que a complexação do $17 \beta$-estradiol com a ciclodextrina tenha causado a maior absorção do hormônio pelos animais, devido a sua capacidade de manter moléculas hidrofóbicas mais biodisponíveis, como visto no trabalho de Paula et al. (2007), no qual, em estudos de solubilidade, a complexação de $\beta$-CD a $17 \beta$ estradiol aumentou a solubilidade aquosa deste, assim como a retenção do fármaco na pele. $\mathrm{O}$ autor atribuiu esse efeito à maior biodisponibilidade do hormônio quando complexado. Observou-se, ao longo do experimento (90 dias), devido ao aumento de exibição dos comportamentos agressivos, um aumento de lesões em alguns indivíduos do grupo BE. No último intervalo de tempo analisado (60-90 dias), também foi observado que os peixes não respondiam mais aos comportamentos agressivos, como visto pela redução significativa $(\mathrm{P}>0,05)$ no ato Afastar (Tab. 2C) dos animais submissos. O comportamento de Nadar rápido para os indivíduos dominantes do grupo $\mathrm{BE}$ apresentou maior frequência (Tab. 2), o que pode estar relacionado ao fato de os outros animais não reagirem ou não serem considerados uma ameaça.

Já no grupo exposto ao $17 \beta$-estradiol $\left(\mathrm{E}_{2}\right)$ livre, houve diminuição da agressividade (zero - 60 dias), conforme visto em alguns comportamentos, como: Perseguição, Fuga e Ataque frontal (Tab. 2), com frequências de exibição inferiores ao grupo controle. Os peixes do grupo E apresentaram o ato de Ficar parado superior se comparado aos demais grupos. Esse mesmo efeito já foi observado em estudo anterior do presente grupo (Santos et al., 2016), em que ocorreu diminuição dos comportamentos agressivos, assim como aumento da inatividade em peixes machos de Betta splendens expostos ao $E_{2}$. Esses resultados corroboram os de Shappell et al. (2010), que também observaram que $\mathrm{o} \mathrm{E}_{2}$, diminuiu a agressividade de Pimephales promelas. Adicionalmente, durante as observações, notou-se o escurecimento corporal dos peixes submissos em todos os grupos experimentais, o que pode estar relacionado a situações de estresse (Volpato et al., 1989). Os mesmos autores relataram ainda outros fatores, como a posse prévia do território e a condição hierárquica anterior, como possíveis explicações para o escurecimento corporal. Esses dois fenômenos também foram observados no presente estudo.

Quanto aos parâmetros zootécnicos, a exposição hormonal não alterou $\mathrm{o}$ ganho de peso e o comprimento dos animais. Foi observado que, durante todo o experimento, os animais se alimentaram normalmente, não havendo diferença estatística $(\mathrm{P}<0,05)$ entre os grupos experimentais. Em relação à sobrevivência dos animais, observou-se uma maior taxa de mortalidade no grupo controle. Raju et al. (2008) sugeriram que o $\mathrm{E}_{2}$ pode apresentar propriedades imunoprotetoras, o que poderia justificar $\mathrm{o}$ observado no presente trabalho. Ainda, a menor taxa de mortalidade foi observada no grupo $\beta$ $\mathrm{CD}: \mathrm{E}_{2}$. A complexação com a ciclodextrina aumenta a estabilidade (Del Valle, 2004; Brewster e Loftsson, 2007) da molécula hospedeira, o que poderia ter potencializado o efeito imunoprotetor do estradiol.

\section{CONCLUSÃO}

Foi possível a construção de um etograma para o estudo comportamental de $O$. niloticus e, sendo o comportamento animal um parâmetro afetado pela presença de substâncias tóxicas, a complexação do $E_{2}$ com a $\beta-C D$ alterou a toxicidade do $E_{2}$, pois aumentou a frequência de exibição principalmente dos comportamentos 
agressivos e também aumentou a sobrevivência dos animais. Não foram observadas alterações em relação ao ganho de peso, à morfometria e ao consumo de alimento entre os grupos estudados. Isso gera a necessidade do desenvolvimento de medidas de controle do descarte dos hormônios complexados a ciclodextrinas no ambiente, pois estes podem apresentar toxicidade diferente de suas formas não complexadas.

\section{AGRADECIMENTOS}

À Universidade Federal Rural de Pernambuco, por permitir o desenvolvimento desse projeto; ao CNPQ (Conselho Nacional de Desenvolvimento Científico e Tecnológico), pelo apoio financeiro (Processo 477215/2013-0).

\section{REFERÊNCIAS}

ALTMANN, J. Observational study of behavior: sampling methods. Behavior, v.48, p.227-267, 1974.

ÁVILA, M.C.; ROMAGOSA, E. Efeito do choque térmico quente em ovos de tilápia nilótica (Oreochromis niloticus): tempo de pósfertilização e duração do processo na sobrevivência das larvas. Bol. Inst. Pesca, v.31, p.55-64, 2005.

BREWSTER, M.E.; LOFTSSON, T. Cyclodextrins as pharmaceutical solubilizers. Adv. Drug Deliver. Rev., v.59, p.645-666, 2007.

CARVALHO, T.B. A interferência $d a$ luminosidade na agressividade e hierarquia social de ciclídeos. 2009. 106f. Tese (Doutorado em Aquicultura) - Centro de Aquicultura, Universidade Estadual de São Paulo, Jaboticabal, SP.

DEL VALLE, E.M.M. Cyclodextrins and their uses: a review. Proc. Biochem., v.39, p.10331046, 2004

DENSLOW, N.; SEPULVEDA, M. Ecotoxicological effects of endocrine disrupting compounds on fish reproduction. In: BABIN, P.J.; CERDA, J.; LUBZENS, E. (Eds.). The fish oocyte - from basic studies to biotechnological applications. Dordrecht: Springer, 2008. p.255322.
FERNANDES, A.N.; GIOVANELA, N.; ALMEIDA, C.A.P. et al. Remoção dos hormônios $17 \beta$-estradiol e $17 \alpha$-etinilestradiol de soluções aquosas empregando turfa decomposta como material adsorvente. Quím. Nova, v.34, p.1526-1533, 2011.

GUIGUEN, Y.; FOSTIER, A.; PIFERRER, F.; CHANG, C.F. Ovarian aromatase and estrogens: a pivotal role for gonadal sex differentiation and sex change in fish. Gen. Comp. Endocrinol., v.165, p.352-366, 2010.

KUBITZA, F. Tilápia: tecnologia e planejamento na produção comercial. Jundiaí: F. Kubitza, 2000. 285p.

LUPI, C.; NHACARINI, N.I.; MAZON, A.F.; SÁ, O.R. Avaliação da poluição ambiental através de alterações morfológicas das brânquias de Oreochromis niloticus (tilápia) nos córregos Retiro, Consulta e Bebedouro, município de Bebedouro-SP. Rev. Fafibe, n.3, p.1-6, 2007.

MAES, J.; VERLOOY, L.; BUENAFE, O.E.; WITTE, M.A.P. et al. Evaluation of 14 organic solvents and carriers for screening applications in zebrafish embryos and larvae. Plos One., v.7, p.1-9, 2012.

MCLACHLAN, J.A.; SIMPSON, E.; MARTIN, $\mathrm{M}$. Endocrine disruptors and female reproductive health. Best Pract. Res. Clin. Endocrinol. Metab., v.20, p.63-75, 2006.

MERIGHE, G.K.F.; PEREIRA, E.M.S.; NEGRÃO, J.A.; RIBEIRO, S. Efeito da cor do ambiente sobre o estresse social em tilápias do Nilo (Oreochromis niloticus). Rev. Bras. Zootec., v.33, p.828-837, 2004.

OISHI, K.; TOYAO, K.; KAWANO, Y. Suppression of estrogenic activity of 17-betaestradiol by beta-cyclodextrin. Chemosphere, v.73, p.1788-1792, 2008.

PAULA, D.; OLIVEIRA, D.C.R.; TEDESCO, A.C.; BENTLEY, M.V.L.B. Efeito promotor de beta-ciclodextrinas modificadas na permeação in vitro do estradiol. Rev. Bras. Cienc. Farm., v.43, p.111-120, 2007.

RAJU, R.; BLAND, K.I.; CHAUDRY, I.H. Estrogen: a novel therapeutic adjunct for the treatment of trauma-hemorrhage-induced immunological alterations. Mol. Med., v.14, p.213-221, 2008. 
SANTOS, B.D.; SILVA, M.C.G.; SANTOS, T.P.; SILVA, S.C.B.L. et al. Efeitos de hormônios esteroides de contraceptivos orais combinados sobre os parâmetros comportamentais de Betta splendens (Regan, 1909). Arq. Bras. Med. Vet. Zootec., v.68, p.387396, 2016.

SEEMANN, F.; KNIGGEB, T.; DUFLOTB, A.; MARIEB, S. et al. Sensitive periods for $17 \beta-$ estradiol exposure during immune system development in seabass head kidney. J. Appl. Toxicol. v.36, p.815-826, 2016.

SHAPPELL, N.W.; HYNDMANB, K.M.; BARTELL, S.E.; SCHOENFUSS, H.L. Comparative biological effects and potency of $17 \alpha$ - and $17 \beta$-estradiol in fathead minnows. Aquat. Toxicol., v.100, p.1-8, 2010.

SPECIES Fact Sheets of Oreochromis niloticus (Linnaeus, 1758), 2016. FAO Available in: $<$ http://www.fao.org/fishery/species/3217/en>. Accessed in: 12 Sep. 2016

TILTON, S.C.; FORAN, C.M.; BENSON, W.H. Relationship between ethinylestradiol-mediated changes in endocrine function and reproductive impairment in Japanese medaka (Oryzias latipes). Environ. Toxicol. Chem., v.24, p.352359,2005
VERA， L.M.; CAIRNS， L.; SÁNCHEZVÁZQUEZ, F.J.; MIGAUD, H. Circadian rhythms of locomotor activity in the Nile Tilapia (Oreochromis niloticus). Chronobiol. Int., v.26, p.666-681, 2009.

VOLPATO, G.L.; FRIOLI, P.M.A.; CARRIERI, M.P. Heterogeneous growth in fishes: some new data in the Nile tilapia Oreochromis niloticus and a general view about the causal mechanisms. Bol. Fisiol. Anim., v.13, p.7-22, 1989.

WU, H.; LI, G.; LIU, S.; HU, N. et al. Monitoring the contents of six steroidal and phenolic endocrine disrupting chemicals in chicken, fish and aquaculture pond water samples using pre-column derivatization and dispersive liquid-liquid microextraction with the aid of experimental design methodology. Food Chem., v.192, p.98-106, 2016.

ZUANON, J.A.S.; SALARO, A.L.; VERAS, G.C.; TAVARES, M.M. et al. Tolerância aguda e crônica de adultos de beta, Betta splendens, à salinidade da água. Rev. Bras. Zootec., v.38, p.2106-2110, 2009. 